\title{
Skin eruption and long-lasting fever in a young man
}

\author{
Marie Minet, ${ }^{1,2}$ Nicolas Hanset, ${ }^{3}$ Jean Cyr Yombi, ${ }^{4}$ Halil Yildiz $^{3}$
}

${ }^{1}$ Emergency medicine, Universite Catholique de Louvain, Bruxelles, Belgium ${ }^{2}$ Department of Pneumologie, Groupement Hospitalierde I'Ouest Lemanique S A, Nyon, Switzerland

${ }^{4}$ Department of Internal Medicine and Perioperative Medicine, Catholic University of Leuven, Brussels, Belgium ${ }^{3}$ Department of Medecine Interne, Cliniques Universitaires Saint-Luc, Bruxelles, Belgium

\section{Correspondence to} Dr Halil Yildiz, halil.yildiz@ uclouvain.be,h_tur.bel@ hotmail.com

Accepted 12 June 2017

\section{CrossMark}

To cite: Minet $\mathrm{M}$, Hanset $\mathrm{N}$, Yombi JC, et al. BMJ Case Rep Published Online First: [please include Day Month Year]. doi:10.1136/bcr-2017220552

\section{DESCRIPTION}

A 20-year-old man presented to emergency room with a 3-week history of general weakness, fever, diffuse arthralgia and skin eruption. His medical history was unremarkable; he did not travel recently, had no contact with an ill person, nor had risky sexual behaviour and took no medication. Physical examination only showed a diffuse, non-painful, infracentimetric and non-confluent macular eruption over the trunks and limbs (figures 1 and 2). Blood test showed elevated $\mathrm{C}$ reactive protein and neutrophilic leucocytosis. Serologies for hepatitis $\mathrm{C}$ and $\mathrm{B}$ viruses, rubella, Epstein-Barr virus, cytomegalovirus, toxoplasmosis and HIV were negative. Urinalysis and chest X-ray were normal. Blood cultures became positive for Neisseria meningitidis, whereas skin biopsy only showed a dermic inflammatory polymorphic (lymphocytic and neutrophilic) infiltrate without specificity with negative aerobic and anaerobic cultures.

A diagnosis of chronic meningococcaemia was retained. We started ceftriaxone $2 \mathrm{~g}$ twice daily with good outcome, and he was discharged from hospital after 7 days of antibiotherapy. The family living under the same roof and the health carriers who were in close contact received one pill of ciprofloxacin $500 \mathrm{mg}$ as secondary prevention.

N. meningitidis is a Gram-negative diplococcus acquired in the nasopharynx through respiratory droplets spread from asymptomatic carrier of a potentially pathogenic strain. Eight serogroups most commonly cause infections in humans (A, B, C, X, Y, Z, W135 and L). Infection can produce a variety of clinical manifestations, ranging from transient fever and bacteraemia to fulminant disease with death ensuing within hours after the onset of clinical symptoms.

Chronic meningococcaemia is defined by a meningococcal bacteraemia with recurrent fever over a period of at least 1 week, without any neurological symptoms or signs of severe sepsis. This chronic evolution of $N$. meningitidis infection is determined by the virulence of the bacteria, host's factors (innate or acquired) or factors influencing the course of the infection (use of non-steroidal anti-inflammatory drug or corticosteroid). This can be encountered at any age, with a higher incidence during adolescence and young adulthood. ${ }^{1}$ Immunologically, properdin deficiency and reduced plasma IgG levels may predispose to chronic meningococcal disease, but the majority of patients (like our patient) with chronic meningococcaemia have a normal humoural immune system. ${ }^{2}$ The classical triad consists of longlasting fever (100\%), skin eruption (100\%, painful in 32\%) and arthralgias (93\%). Myalgias (30\%) and headache (without meningeal syndrome) may be present. In $60 \%$ of cases, general state is good.

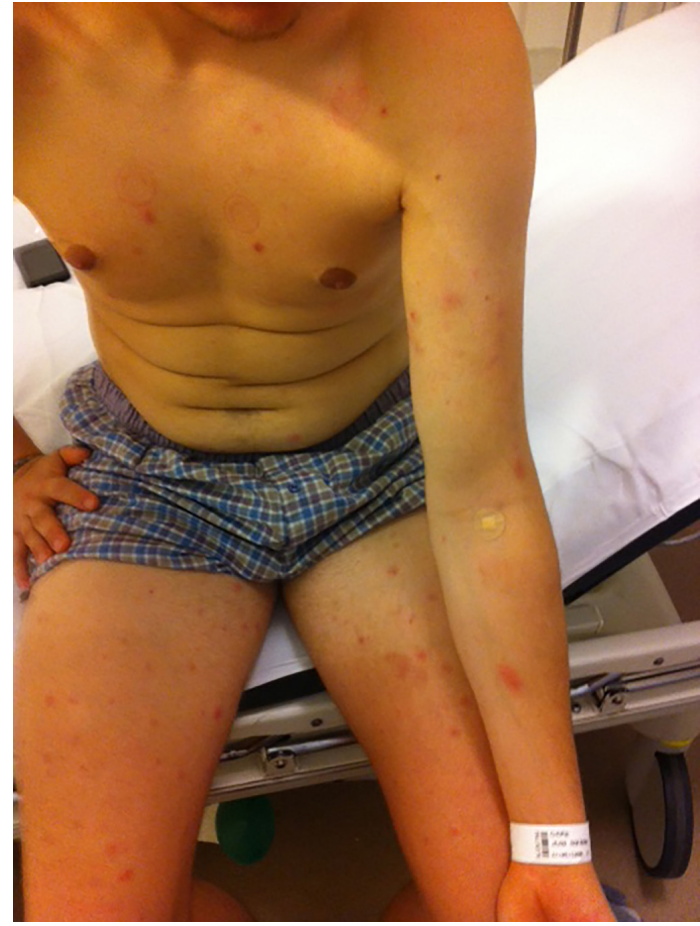

Figure 1 Macular lesions on the trunk.

Lumbar puncture is not useful for the diagnosis. Chronic infection concerns the B serotype in 60\% of cases but in our patient, it was a serotype C. A rapid diagnosis is important to avoid complications such as secondary infection (meningitis, glomerulonephritis and epididymitis), contagion and death. Symptoms are often rapidly degressive with antibiotics. Penicillin alone is the recommended treatment (4 million units intravenously every 4 hours), but third-generation cephalosporin (ceftriaxone intravenously 2 g every 12 hours) may be used due to the simplicity of the treatment ${ }^{3}$ or while the susceptibility to penicillin is not yet confirmed. There is no consensus about the duration of the antibiotherapy, but 7 days of therapy would usually be sufficient accordingthe response of the patient.

Contributors MM and HY contributed equally to the writing of this paper. JCY and NH helped for writing the paper and management of the patient.

Competing interests None declared.

Patient consent Obtained.

Provenance and peer review Not commissioned; externally peer reviewed.

(c) BMJ Publishing Group Ltd (unless otherwise stated in the text of the article) 2017. All rights reserved. No commercial use is permitted unless otherwise expressly granted. 


\section{Images in...}

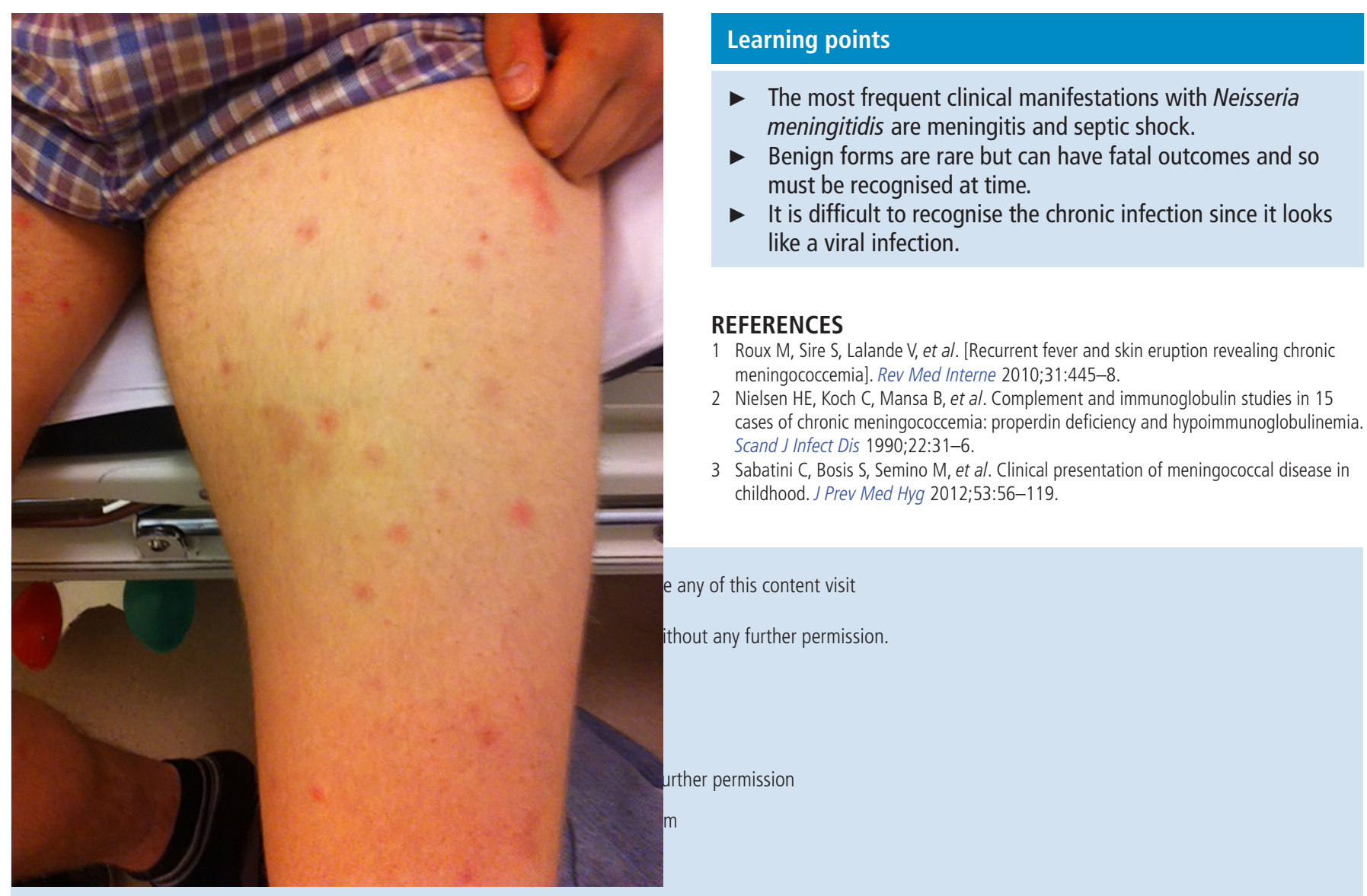

Figure 2 Macular lesions on the left limb. 